\title{
Ocorrência de dor em bebês internados em unidade de terapia intensiva neonatal de maternidade terciária
}

\section{Pain occurrence in interned babies in neonatal intensive care unit at a tertiary maternity}

\author{
Caroline Diniz Nazareth ${ }^{1}$. Maria Francielze Holanda Lavor ${ }^{2}$. Tânia Maria Araújo Santos Sousa ${ }^{3}$. \\ 1 Médica pediatra, concludente da Residência Médica em Neonatologia da Maternidade-Escola Assis Chateaubriand. 2 Médica \\ pediatra e neonatologista, Mestra em Saúde Pública pela Universidade Federal do Ceará, Supervisora do Programa de \\ Residência Médica em Neonatologia da Maternidade-Escola Assis Chateaubriand da Universidade Federal do Ceará. 3 Médica \\ pediatra e neonatologista, Mestra em Saúde da Criança e do Adolescente pela Universidade Estadual do Ceará, Médica \\ assistente de Unidade Neonatal da Maternidade-Escola Assis Chateaubriand da Universidade Federal do Ceará.
}

\section{RESUMO}

Introdução: Por muitos anos, acreditou-se que os recém-nascidos (RNs) eram incapazes de sentir dor, devido ao pressuposto que seu sistema nervoso ainda não estaria completamente formado. Os RNs podem perceber a dor com mais intensidade do que as crianças e os adultos, devido aos seus mecanismos de controle inibitório imaturos, diminuindo capacidade de modular a experiência dolorosa. Objetivos: Identificar a ocorrência de dor em bebês internados e mensurar a utilização de analgesia farmacológica. Material e Métodos: Estudo transversal prospectivo, realizado nas Unidades de Terapia Intensiva Neonatal de um hospital terciário, no período de novembro de 2014 a fevereiro de 2015. Resultados: Dor foi identificada em 36\% dos pacientes. Cinco (10\%) pacientes receberam medidas farmacológicas de analgesia. Conclusão: Houve discrepância entre a frequência de dor encontrada e a de utilização de medidas para aliviá-la. Mostra-se necessária a implantação de um protocolo de avaliação e terapêutica de dor pela Instituição.

Palavras-chave: Recém-nascido. Dor. Medição da dor. Unidades de terapia intensiva neonatal.

\section{ABSTRACT}

Introduction: For many years, it has been believed that newborns weren't able to feel pain, because their nervous system wasn't completely built. Newborns feel more pain than child and adults, due to the lack of maturity of the descendent inhibitory pathways, decreasing their ability to modulate pain experience. Objective: To identify occurrence of pain in interned babies and measure the use of pharmacological analgesia. Methods: Transversal prospective study, performed at Neonatal Intensive Care Units (NICUs) of tertiary hospital, between November 2014 and February 2015. Results: Pain was identified in 36\% of patients. Five (10\%) patients received pharmacological analgesia. Conclusion: A discrepancy arises between frequency of pain and the use of relieving measures. It is necessary to implement a protocol of evaluation and pain relief at the Institution.

Keywords: Infant, newborn. Pain. Pain measurement. Intensive care units, neonatal.

Autor correspondente: Caroline Diniz Nazareth. Rua Coronel Nunes de Melo, s/n, Rodolfo Teófilo, Fortaleza, Ceará. CEP:60430-270. Telefone: +55 85 3366-8528. E-mail: carolitaped@yahoo.com.br.

Conflito de interesses: Não há qualquer conflito de interesses por parte de qualquer um dos autores.

Recebido em: 25 Mai 2015; Revisado em: 18 Jun 2015; Aceito em: 22 Jun 2015. 


\section{INTRODUÇÃO}

A dor é uma experiência complexa que não envolve apenas a transmissão de estímulos nociceptivos, mas também o processamento nas dimensões cognitiva e afetiva pelo córtex cerebral. ${ }^{1}$ Por muitos anos, acreditou-se que os recémnascidos (RNs) eram incapazes de sentir dor, devido ao pressuposto que seu sistema nervoso ainda não estaria completamente formado. ${ }^{2,3}$

As vias anatômicas responsáveis pela dor (neurotransmissores, ramificações dendríticas e talâmicas) encontram-se desenvolvidas de forma precoce na sétima semana de gestação e totalmente distribuídas na superfície corporal em torno da vigésima semana de gestação. ${ }^{1,4}$ De fato, com 24 semanas de gestação estímulos dolorosos estão associados com marcadores fisiológicos, metabólicos e hormonais de resposta ao stress. ${ }^{1,5}$ A mielinização das vias nociceptivas completa-se em torno de 30 semanas de gestação. ${ }^{1,4}$

Os RNs podem perceber a dor com mais intensidade do que as crianças e os adultos, visto que as vias anatômicas, neurofisiológicas e hormonais já estão prontas ao nascimento, enquanto as vias capazes de inibi-la e reduzi-la não estão. ${ }^{2,6}$ Isso diminui a capacidade de modular a experiência dolorosa. ${ }^{1}$ A percepção da dor mostra-se ainda mais exacerbada em prematuros, como consequência da maior imaturidade das vias medulares descendentes inibitórias. $^{7}$

O desenvolvimento das unidades de terapia intensiva neonatais tem proporcionado uma diminuição da mortalidade de recém-nascidos gravemente enfermos. Paralelamente à sofisticação dos recursos terapêuticos, um maior número de exames e procedimentos invasivos é necessário para garantir a sobrevivência desses neonatos. A sobrevivência ao período neonatal tem um custo para o paciente, que inclui a dor. ${ }^{8}$ Calcula-se que cada recém-nascido (RN) internado em Unidade de Terapia Intensiva Neonatal (UTIN) receba cerca de doze procedimentos dolorosos ao dia durante as duas primeiras semanas de internação, a maioria sem qualquer analgesia. $^{7}$

A subjetividade envolvida na avaliação do evento doloroso nessa faixa etária caracterizada pela incapacidade do neonato em verbalizar aliada à falta de treinamento profissional específico dificultam o diagnóstico e o tratamento adequado da dor neonatal na prática diária. ${ }^{5,9}$ Existem várias escalas que ajudam na identificação da dor ou do desconforto, facilitando intervenções específicas. ${ }^{10}$

Diante da necessidade pungente de reconhecer e tratar a dor no recém-nascido, este artigo visa a identificar a ocorrência de dor em bebês internados em UTI neonatal, através da aplicação de duas escalas, assim como mensurar a utilização de analgesia farmacológica.

\section{MATERIAL E MÉTODOS}

Trata-se de um estudo transversal prospectivo, realizado nas Unidades de Terapia Intensiva Neonatal (UTIN) da
Maternidade-Escola Assis Chateaubriand (MEAC). A MEAC possui duas UTINs separadas apenas pela estrutura física do hospital, cujo critério de internação em cada unidade é unicamente a disponibilidade de leito.

O protocolo de pesquisa foi aprovado pelo Comitê de Ética em Pesquisa da instituição, estando de acordo com os critérios estabelecidos pela Declaração de Helsinki com as suas modificações. Não houve necessidade de consentimento informado por parte dos familiares, pois se tratou de levantamento de dados populacionais de neonatos internados nas referidas unidades, mas foi garantida a confidencialidade das informações obtidas de cada paciente e de cada unidade.

A pesquisa foi realizada no período de novembro de 2014 a fevereiro de 2015. O único critério de inclusão foi estar internado em uma das duas unidades neonatais universitárias no período do estudo. Assim, foi incluído todo paciente internado por qualquer período (horas ou dias) ou por qualquer motivo, segundo a definição de cuidados intensivos do hospital. Dessa forma, não foi necessário calcular o poder amostral, pois não foi avaliada uma amostra da população, mas todos os pacientes internados durante o período escolhido para a coleta de dados, realizada entre os meses de dezembro de 2014 e janeiro de 2015. Foram excluídos do estudo os pacientes com escore de Apgar no quinto minuto menor que sete. Também foi excluído do estudo um paciente com síndrome de Moebius, em decorrência na inabilidade de apresentar expressões faciais. Dos 55 pacientes avaliados, cinco foram excluídos, permanecendo, assim, 50 para avaliação estatística.

Foi realizada a análise de prontuários para a coleta de dados clínicos e epidemiológicos dos pacientes e aplicadas escalas de dor. Duas escalas de dor foram aplicadas a todos os pacientes: Sistema de Codificação da Atividade Facial Neonatal (Neonatal Facial Coding System - NFCS) e Escala de Dor no Recém-Nascido e no Lactente (Neonatal Infant Pain Scale - NIPS). Estas escalas são validadas por estudos internacionais. A aplicação das escalas e a coleta dos dados foram realizadas por uma única pessoa, a autora principal, eliminando, assim, qualquer heterogeneidade de avaliação subjetiva.

Os dados foram analisados no software Epi Info versão 3.5.2. e analisados com o auxílio do software SPSS. Para a análise estatística utilizaram-se métodos descritivos, sendo as variáveis numéricas agrupadas em média, enquanto as qualitativas serão agrupadas em percentuais. Para análise dos dados a frequência dos achados será agrupada em prevalência e serão feitas porcentagens simples em relação ao total de pacientes.

\section{RESULTADOS}

Participaram do estudo 50 pacientes, sendo 19 (38\%) do sexo feminino e 31(62\%), do masculino. A média de peso ao nascer foi de $1896 \mathrm{~g}$. A variação foi de $590 \mathrm{~g}$ a $3650 \mathrm{~g}$. Dezenove pacientes pesavam menos que $1500 \mathrm{~g}$. A maior parte dos pacientes nasceu com idade gestacional entre $31 \mathrm{e}$ 
34 semanas, variando entre 26 a 40 semanas, conforme descrito na Tabela 1. A avaliação foi realizada em pacientes com idade de um dia de vida até 189 dias, com mediana de idade de dois dias e média de 14 dias de vida. Quatro pacientes não se encontravam no período neonatal, ou seja, possuíam mais de 28 dias de vida. A idade deles variou de 33 a 189 dias. Esses pacientes foram mantidos no estudo em decorrência da relevância dos dados obtidos através de sua avaliação. O escore de Apgar no quinto minuto de vida foi, em média, de oito, variando de sete a nove.

Tabela 1. Distribuição da presença de dor por idade gestacional em uma amostra de pacientes internados em UTI neonatal, na Maternidade-Escola Assis Chateaubriand, Fortaleza, Ceará, 2015.

\section{IDADE $\mathbf{N}$ \\ DOR / \% \\ GESTACIONAL}

\begin{tabular}{lll}
\hline$<\mathbf{2 4}$ semanas & 0 & 0 \\
$\mathbf{2 4}$ - $\mathbf{3 0}$ semanas & 14 & $7(50 \%)$ \\
$\mathbf{3 1}$ - $\mathbf{3 4}$ semanas & 19 & $6(31,6 \%)$ \\
$\mathbf{3 5}$ - $\mathbf{3 7}$ semanas & 8 & $4(50 \%)$ \\
$>\mathbf{3 7}$ semanas & 9 & $1(11,1 \%)$ \\
TOTAL & $\mathbf{5 0}$ & $\mathbf{1 8}$ \\
\hline
\end{tabular}

Seis (12\%) apresentavam malformações congênitas. As malformações identificadas foram: defeito do septo atrioventricular, pé torto congênito, ventrículo único, gastrosquize e coarctação de aorta. Três pacientes com malformação foram excluídos do estudo: um com síndrome de Edwards, outro com comunicação interatrial, e ainda um com síndrome de Moebius.

Seis $(12 \%)$ pacientes usavam anticonvulsivantes. Cinco $(10 \%)$ deles utilizavam fenobarbital, sendo que destes um (2\%) usava também fenitoína. O uso de anticonvulsivantes, assim como o de sedativos, pode subestimar a presença de dor nos pacientes por diminuir as respostas esperadas aos estímulos nociceptivos. ${ }^{4,12}$

Durante a avaliação, quatro (8\%) recém-nascidos foram submetidos a procedimentos dolorosos. Os procedimentos foram: punção venosa para coleta de exames, punção venosa para obtenção de acesso periférico, aferição da pressão arterial em membro superior e reanimação cardiopulmonar (massagem cardíaca externa associada à ventilação com pressão positiva com bolsa-valva- cânula orotraqueal). Nenhum destes recebeu analgesia específica para a realização destes procedimentos dolorosos.

Apenas seis (12\%) pacientes haviam sido submetidos a procedimentos cirúrgicos. As cirurgias foram: ligadura do canal arterial (dois pacientes), laparotomia exploradora, gastrostomia com fundoplicatura à Nissen, correção de gastrosquize e dissecção de veia jugular externa. Os pacientes submetidos à correção de gastrosquize e à gastrostomia foram avaliados no primeiro dia de pós- operatório. O paciente da laparotomia foi avaliado no sétimo dia após a cirurgia. Os demais foram avaliados tardiamente, mais de duas semanas após os procedimentos.

Dor foi identificada em dezoito (36\%) pacientes, conforme apresentado na Tabela 2. Na escala NFCS, a dor mostrou-se presente em $15(30 \%)$ pacientes. Dezessete (34\%) apresentaram dor na escala NIPS. Dor foi incidente nas duas escalas em 14 (28\%) pacientes.

Tabela 2. Identificação de dor em uma amostra de pacientes internados em UTI neonatal, na Maternidade-Escola Assis Chateaubriand, Fortaleza, Ceará, 2015.

\begin{tabular}{lcc}
$\begin{array}{l}\text { IDENTIFICAÇÃO DE } \\
\text { DOR }\end{array}$ & N & \% \\
\hline $\begin{array}{l}\text { DOR EM PELO MENOS } 1 \\
\text { ESCALA }\end{array}$ & 18 & $36 \%$ \\
DOR NA ESCALA NFCS & 17 & $34 \%$ \\
DOR NA ESCALA NIPS & 15 & $30 \%$ \\
$\begin{array}{l}\text { DOR NAS DUAS } \\
\text { ESCALAS }\end{array}$ & 14 & $28 \%$ \\
\end{tabular}

Cinco (10\%) pacientes receberam medidas farmacológicas de analgesia. Os cinco utilizaram fentanil; um deles utilizou além de fentanil, tramadol e paracetamol. Este era o paciente no sétimo dia pós-operatório de laparotomia exploradora, para o qual foi prescrito a realização de avaliação de dor, através da escala NFCS, de 3 em 3 horas. Os pacientes avaliados no primeiro dia pós-cirurgia não receberam qualquer tipo de analgesia, além disso, não estavam sendo avaliados com escala de dor no dia da coleta dos dados. A Tabela 3 revela a presença de dor e a utilização de analgesia medicamentosa em pacientes cirúrgicos.

Tabela 3. Identificação de dor e de analgesia medicamentosa em uma amostra de pacientes cirúrgicos internados em UTI neonatal, na Maternidade-Escola Assis Chateaubriand, Fortaleza, Ceará, 2015.

\begin{tabular}{ccc}
$\begin{array}{c}\text { PACIENTES } \\
\text { EM PÓS- } \\
\text { OPERATÓRIO }\end{array}$ & $\begin{array}{c}\text { IDENTIFICADA } \\
\text { DOR }\end{array}$ & $\begin{array}{c}\text { ANALGESIA } \\
\text { FARMACOLÓGICA }\end{array}$ \\
\hline $5(100 \%)$ & $1(20 \%)$ & $2(40 \%)$ \\
\hline
\end{tabular}

Observaram-se medidas não farmacológicas de analgesia na avaliação de dois pacientes (4\%). A medida adotada foi a sucção não-nutritiva, sendo realizada como mecanismo de conforto em dois pacientes os quais não estavam sendo submetidos a procedimentos dolorosos naquele momento.

\section{DISCUSSÃO}

Na UTIN há predomínio de situações adversas decorrentes tanto das normas e rotinas institucionais, quanto do complexo processo de trabalho, as quais contribuem para o 
desarranjo da homeostasia do organismo do recém-nascido. Dentre elas, destacam-se o ambiente com luminosidade e temperatura artificial, o barulho incômodo e estressante e ainda, a quantidade de manipulações realizadas nestes pacientes, em geral, agressivas e dolorosas. A associação da dor causada por esses procedimentos com o ambiente estressor poderão impactar em sua qualidade de vida e desenvolvimento neuropsicomotor. ${ }^{12}$

Estímulos dolorosos agudos desencadeiam nos recémnascidos uma resposta global ao estresse que inclui modificação a nível cardiovascular, respiratório, imunológico, hormonal e comportamental. O desequilíbrio na fisiologia do organismo pode acarretar queda na saturação de oxigênio, aumento das frequências cardíaca e respiratória, além de consequências em longo prazo, como o comprometimento do crescimento, desenvolvimento, diminuição do limiar de dor, hiperalgia e alodinia. ${ }^{1,10,12}$

$\mathrm{O}$ fato de a dor ser um fenômeno subjetivo gera uma grande dificuldade para a elaboração de um método único de avaliação e de fácil aplicação na prática clínica dos profissionais das unidades neonatais. ${ }^{10}$

Foi de especial importância a padronização da dor como quinto sinal vital, pela Joint Commission on Accreditation of Healthcare Organizations (JCAHO). Esta comissão passou a considerar prioritárias a avaliação, a intervenção e a reavaliação da dor no processo de qualificação hospitalar. ${ }^{12}$

Há evidências científicas de que o recém-nascido apresenta um modo característico de exprimir a dor, através de uma linguagem própria, sendo importante neste processo a adoção das escalas. A implementação das escalas de avaliação da dor em recém-nascidos mostra-se essencial, como uma ferramenta clínica de baixo custo e de alto impacto na identificação deste fenômeno. ${ }^{13,14}$ Ressalta-se que ainda não existe uma escala padrão ouro para avaliação da dor no RN. Com base no conhecimento das características de cada escala, não se pode eleger a mais adequada, pois a escolha dependerá da idade gestacional, do tipo de estímulo doloroso, do contexto em que o RN se apresente. ${ }^{13}$

A observação da expressão facial é um método não invasivo de avaliação de dor, sensível e útil na clínica diária. Trata-se também de um método específico para avaliação da dor em recém-nascidos prematuros e de termo. Várias formas de avaliação da expressão facial foram desenvolvidas para o estudo objetivo da dor no neonato. Dentre elas, destaca-se o Sistema de Codificação da Atividade Facial Neonatal NFCS. Sabe-se que fronte saliente, olhos espremidos, sulco nasolabial aprofundado e lábios entreabertos estão presentes em mais de 90\% dos recém-nascidos submetidos a um estímulo doloroso. Além de ser confiável, é de fácil aplicabilidade e pode ser realizada à beira do leito8. Na escala NFCS, considera-se a presença de dor quando três ou mais movimentos faciais aparecem de maneira consistente durante a avaliação da presença de dor. Seu escore máximo é de oito pontos. ${ }^{8,10,15}$

A Escala de dor no recém-nascido e no lactente (Neonatal Infant Pain Scale - NIPS) é composta por cinco indicadores de dor comportamentais e um fisiológico. Trata-se de uma escala válida, pois se baseia nas alterações comportamentais frente à dor descritas na literatura. A NIPS tem se mostrado útil para a avaliação de dor em neonatos a termo e prematuros, conseguindo diferenciar os estímulos dolorosos dos não dolorosos. A pontuação varia de zero a sete, definindo-se presença de dor valores iguais ou superiores a quatro. ${ }^{8,15}$

Neste estudo, as escalas NFCS e NIPS mostraram-se equivalentes na percepção de dor nos pacientes, dado consistente com a literatura atual. ${ }^{8,15}$ Reforça-se, portanto, a confiabilidade das escalas utilizadas.

Embora o alívio da dor seja um dos princípios básicos da medicina, na prática, a analgesia em pacientes com dificuldade para verbalizar sensações e sentimentos é frequentemente ignorada. Desde 1970, numerosos estudos têm demonstrado que o subtratamento da dor é comum, especialmente em crianças e recém-nascido. A dificuldade para reconhecer e avaliar a dor no período neonatal constitui um dos maiores obstáculos ao seu tratamento adequado nas unidades de terapia intensiva. A falta de conhecimento a respeito das escalas utilizadas para avaliar a dor, das indicações para uso de analgésicos e de seus efeitos colaterais nessa faixa etária contribui para essa realidade. ${ }^{16}$

Vale enfatizar a dificuldade da incorporação prática de conhecimentos já solidificados no que se refere à presença e às consequências da dor para o recém-nascido. Há necessidade de aperfeiçoar a formação dos profissionais de saúde para diminuir a distância entre os conhecimentos existentes a respeito do manejo da dor no período neonatal e a prática clínica. ${ }^{16}$

Em primeiro lugar, é importante tentar minimizar as agressões sofridas pelo recém-nascido durante a sua permanência nas unidades de terapia intensiva. Assim, devese controlar a incidência de luzes fortes sobre a criança, tentar diminuir o ruído à sua volta e racionalizar a manipulação do paciente, de tal modo que os cuidados apropriados sejam realizados, mas que se preserve períodos livres para o sono. ${ }^{8,15}$ Além disso, considera-se importante inserir os pais no ambiente da UTIN. A neurociência mostra que uma ligação forte e segura com os pais parece ter uma função biológica protetora, resguardando o bebê dos efeitos da dor e do estresse. ${ }^{17}$

Existem medidas farmacológicas e não-farmacológicas para alívio da dor em recém-nascidos. ${ }^{6,8}$ As farmacológicas incluem medicações como os antiinflamatórios não hormonais e os opióides. Os métodos não farmacológicos podem envolver estímulos visuais, auditivos, táteis e gustativos. $^{15}$

A pequena frequência de emprego de medidas farmacológicas para alívio da dor encontra-se em consonância com a literatura. Estes estudos enfatizam o fato de, até mesmo procedimentos considerados bastante dolorosos, como a intubação orotraqueal, são realizados eletivamente sem analgesia. ${ }^{4,10}$ Há evidências de que a intubação orotraqueal sem analgesia em neonatos pode levar 
a complicações hemodinâmicas como hipoxemia, bradicardia, hipertensão intracraniana e hemorragia periintraventricular. ${ }^{18,19}$

Nas unidades de terapia intensiva neonatais, o uso de analgesia e sedação é eventual, desconsiderando-se a dor e o desconforto do paciente, que ainda não apresenta condições verbais de expor o que sente. Apesar dos avanços no conhecimento da fisiologia da dor nessa faixa etária, do desenvolvimento de métodos de avaliação da dor para o lactente pré-verbal e da existência de medidas terapêuticas para o alívio da dor, ainda há uma grande lacuna entre o conhecimento teórico e a conduta prática por parte dos profissionais de saúde. ${ }^{8}$

Percebeu-se que na instituição onde se realizou o estudo ainda não se utilizam rotineiramente escalas para se mensurar a dor. Não existe até o momento, um protocolo de como identificar e aliviar a dor nos pacientes internados. Alguns estudos revelam que, mesmo em UTINs onde há escalas determinadas para avaliar a dor no recém-nascido, essa avaliação ou não é realizada, ou não é levada em

\section{REFERÊNCIAS}

1. Simons SH, Tibboel D. Pain perception development and maturation. Semin Fetal Neonatal Med. 2006;11(4):227-31.

2. Alencar AJ. Como o bebê vê. Revista de Pediatria do Ceará. 2009;10:53-7.

3. Mendes LC, Fontenele FC, Dodt RC, Almeida LS, Cardoso MV, Silva CB. A dor no recém-nascido na Unidade de Terapia Intensiva Neonatal. Rev Enferm UFPE on line. 2013;7(11):6446-54.

4. Guaragni B, Howell A, Rehman FU, Jain A. Management of pain in ventilated neonates: current evidence. Paediatr Child Health. 2014;24(1):32-7.

5. Maxwell LG, Malavolta CP, Fraga MV. Assessment of pain in the neonate. Clin Perinatol. 2013;40(3):457-69.

6. Carbajal R, Rousset A, Danan C, Coquery S, Nolent P, Ducrocq $\mathrm{S}$, et al. Epidemiology and treatment of painful procedures in neonates in intensive care units. JAMA. 2008;300(1):60-70.

7. Grunau RE, Holsti L, Peters JW. Long-term consequences of pain in human neonates. Semin Fetal Neonatal Med. 2006;11(4):268-75.

8. Guinsburg R. Avaliação e tratamento da dor no recém-nascido. J pediatr (Rio J). 1999;75(3):149-60.

9. Scherman E, Johansson L, Gradin M, van den Berg J, Eriksson M. Procedural pain in neonates: do nurses follow national guidelines? A survey to Swedish neonatal units. Journal of Neonatal Nursing. 2014;20(1):31-6.

10. Arias MC, Guinsburg R. Differences between uni-and multidimensional scales for assessing pain in term newborn infants at the bedside. Clinics (Sao Paulo). 2012;67(10):1165-70. consideração pelos profissionais. Destaca-se a necessidade imperiosa dos serviços possuírem, utilizarem e atualizarem as rotinas e protocolos para a avaliação e tratamento da dor no $\mathrm{RN}$, assim como o treinamento e capacitação dos profissionais que trabalham nestas unidades, garantindo a aplicação na prática dos conhecimentos relacionados à prevenção, avaliação e tratamento da dor, com objetivo de uniformizar a atuação dos profissionais do serviço e permitir um tratamento adequado aos RN.

\section{CONCLUSÃO}

Dor revelou-se presente em 36\% dos pacientes analisados. As escalas aplicadas mostraram-se equivalentes para identificar dor. Apenas $10 \%$ dos pacientes receberam analgesia medicamentosa. Houve, portanto, uma discrepância entre a frequência de dor encontrada e a de implementação de medidas para aliviá-la. Mostra-se necessária criação de um protocolo de avaliação e de terapêutica da dor dos pacientes internados nas Unidades de Terapia Intensiva Neonatais da Instituição.

11. Serpa AB, Guinsburg R, Balda RC, Santos AM, Areco KC, Peres CA. Multidimensional pain assessment of preterm newborns at the $1^{\text {st }}, 3^{\text {rd }}$ and $7^{\text {th }}$ days of life. Sao Paulo Med J. 2007;125(1):2933.

12. Santos LM, Pereira MP, Santos LF, Santana RC. Avaliação da dor no recém-nascido prematuro em Unidade de Terapia Intensiva. Rev Bras Enferm. 2012;65(1):27-33.

13. Melo GM, Lélis AL, Moura AF, Cardoso MV, Silva VM. Escalas de avaliação de dor em recém-nascidos: revisão integrativa. Rev Paul Pediatr. 2014;32(4):395-402.

14. Santos LM, Ribeiro IS, Santana RC. Identificação e tratamento da dor no recém-nascido prematuro na Unidade de Terapia Intensiva. Rev Bras Enferm. 2012;65(2):269-75.

15. Parry S. Acute pain management in the neonate. Anaesthesia and Intensive Care Medicine. 2014;15(3):111-15.

16. Prestes AC, Guinsburg R, Balda RC, Marba ST, Rugolo LM, Pachi PR, et al. Freqüência do emprego de analgésicos em unidades de terapia intensiva neonatal universitárias. J Pediatr (Rio J). 2005;81(5):405-10

17. Alencar AJ, Rolim KM. Bases científicas do acolhimento amoroso ao recém-nascido. Rev Pediatr Ceará. 2006;7(1):27-32.

18. Badiee Z, Vakiliamini M, Mohammadizadeh M. Remifentanil for endotracheal intubation in premature infants: a randomized controlled trial. J Res Pharm Pract. 2013; 2(2):75-82.

19. Mosalli R, Shaiba L, Alfaleh K, Paes B. Premedication for neonatal intubation: current practice in Saudi Arabia. Saudi J Anaesth. 2012;6(4):385-92.

\section{Como citar:}

Nazareth CD, Lavor MF, Sousa TM. Ocorrência de dor em bebês internados em unidade de terapia intensiva neonatal de maternidade terciária. Rev Med UFC. 2015 jan-jun;55(1):33-37. 